BMJ Open

Diabetes

Research

\& Care

\section{Caucasian and Asian difference in role of type 1 diabetes on large-for- gestational-age neonates}

To cite: Guo Y, Luo R, Corsi DJ, et al. Caucasian and Asian difference in role of type 1 diabetes on large-forgestational-age neonates BMJ Open Diab Res Care 2020;8:e001746. doi:10.1136/ bmjdrc-2020-001746

- Supplemental material is published online only. To view please visit the journal online (http://dx.doi.org/10.1136/ bmjdrc-2020-001746).

YG and SWW contributed equally.

Received 1 July 2020

Revised 10 September 2020

Accepted 26 0ctober 2020

\section{ABSTRACT}

Introduction Racial differences in the association between type 1 diabetes mellitus (T1DM) and largefor-gestational-age (LGA) neonates remain unclear. The objective of this study was to compare the effect of T1DM on LGA neonates between Caucasian and Asian women. Research design and methods A population-based retrospective cohort study was conducted among Caucasian and Asian women who had prenatal screening and gave a singleton live birth in an Ontario hospital between April 2015 and March 2018. Multivariable logbinomial regression models were used to estimate the adjusted relative risks (aRRs) and 95\% Cls of T1DM on LGA for Caucasian and Asian women. Relative contribution of T1DM to LGA was examined by multivariable logistic regression model, stratified by Caucasian and Asian women.

Results A total of 232503 women (69.4\% Caucasians and $30.6 \%$ Asians) were included in the final analysis. The rate of T1DM was higher in Caucasians $(0.5 \%)$ than in Asians $(0.2 \%)$, and the rate of LGA neonates was also higher in Caucasians (11.0\%) than in Asians (5.0\%). The association between T1DM and LGA in Caucasians (aRR $4.18,95 \% \mathrm{Cl}$ (3.84 to 4.55)) was more robust than that in Asians (aRR 2.11, 95\% Cl (1.24 to 3.59)). T1DM was the fourth strongest contributor to LGA in Caucasians, while T1DM was the seventh contributor to LGA in Asians. Conclusions T1DM plays a more substantial role in LGA among Caucasians than Asians. Clinicians should be aware of the Caucasian-Asian differences of effects of T1DM on LGA when developing pregnancy management strategies.

\section{INTRODUCTION}

Type 1 diabetes mellitus (T1DM) is an autoimmune disease characterized by selective destruction of the insulin-secreting $\beta$ cells, making up an estimated $5 \%-10 \%$ ofall diabetes cases. ${ }^{1}$ It is well recognized that women with T1DM have two to five times higher risk of large-for-gestational-age (LGA) neonates, resulting in short-term and long-term adverse maternal and offspring outcomes. The shortterm complications include prolonged labor, third-degree and fourth-degree perineal tears, postpartum hemorrhage, and cesarean section, neonatal hypoglycemia, respiratory disorders, hyperbilirubinemia, shoulder

\section{Significance of this study}

What is already known about this subject?

- Incidence of type 1 diabetes mellitus (T1DM) is substantially higher among Caucasians than Asians.

- Maternal prepregnancy obesity, excessive gestational weight gain, and gestational diabetes are welldocumented risk factors for large-for-gestational age (LGA) across different race groups, but the racial differences in the association between T1DM and LGA remain unclear.

What are the new findings?

- Rates of T1DM and LGA were higher in Caucasians than in Asians.

- Significant modification effect of race on the association between T1DM and LGA was observed, with stronger association of T1DM with LGA in Caucasians than in Asians.

- T1DM was the fourth strongest contributor to LGA in Caucasians, while it was the seventh contributor to LGA development in Asians.

How might these results change the focus of research or clinical practice?

- Further research on better understanding the etiology of Caucasian-Asian differences in effect of T1DM on LGA would be of value to inform clinical practices on prenatal management to possibly prevent T1DMassociated morbidity in the offspring.

dystocia, and admission to the neonatal intensive care unit. ${ }^{2-4}$ In addition, infants with LGA have an increased lifetime risk of obesity, type 2 diabetes mellitus (T2DM), and chronic diseases. $^{5}$

The incidence of T1DM has been increasing by $2 \%-5 \%$ worldwide in the past decade, with approximately 78000 youth newly diagnosed annually. ${ }^{67}$ In the USA, the number of people affected by T1DM is estimated to be up to three million. ${ }^{7}$ In Canada, about 300000 Canadians live with T1DM, and the annual incidence rate has been growing at an estimated $5.1 \%$ which is higher than the global average. ${ }^{8}$ It has been reported that there were 
significant racial differences in the incidence of T1DM between Caucasians and Asians below the age of 15 years old, with a high incidence rate above 20 cases per 100000 per year in Caucasians, while a rate of less than 1 case per 100000 individuals per year has been reported in some Asian countries. ${ }^{9}$

Appropriate glycemic control before and during pregnancy is believed to be the basis for improved pregnancy outcome among women affected by T1DM. Unfortunately, the rate of fetal overgrowth remains significant at around 50\% among pregnancies with T1DM, even though many advances in diabetes management and therapies have been applied, ${ }^{10}$ suggesting that other contributors may be involved. In addition to T1DM, maternal prepregnancy obesity, excessive gestational weight gain, gestational diabetes, and T2DM are known as the main risk factors of LGA. ${ }^{11}{ }^{12}$ Several studies showed racial differences in effects of prepregnancy obesity, gestational weight gain, and gestational diabetes on LGA, ${ }^{1314}$ but the Caucasian-Asian disparities in the association between T1DM and LGA were not reported.

The mechanisms of Caucasian-Asian differences in T1DM remain unclear. Clinical and immunologic characteristics of T1DM in Asian populations are different from those of Caucasians. ${ }^{9}$ Furthermore, the underlying pathophysiology of T1DM in pregnancy and risk of LGA neonates between Caucasians and Asians might be fundamentally different due to differences in genetic and environmental factors. ${ }^{6}$ Some investigators have suggested that the immune attack at insulin-secreting beta cells as the pathogenesis of T1DM may involve polygenic factors and environmental triggers. ${ }^{15}$ The primary genetic susceptibility of T1DM has been reported to be attributable to the high-risk human leukocyte antigen (HLA) genotypes and haplotypes, which present less frequently in Asians. ${ }^{9}$ It potentially leads to less severe T-cell-mediated immune destruction of beta cells in the pancreatic islets of Langerhans in Asians than in Caucasians, with lower level of islet-specific autoantibodies. ${ }^{9}$ Racial differences in dietary protein intake, gut microbiota, chemical toxin exposure, ${ }^{16-18}$ and viral or bacterial infections ${ }^{15}$ may also lead to heterogeneous autoimmunologic pathways in destroying beta cells between Caucasians and Asians. Thus, it is reasonable to assume that the role of T1DM on LGA may be different between Caucasians and Asians.

The current study aims to examine the CaucasianAsian differences in the association between T1DM and LGA neonates, including the relative contribution of T1DM to neonatal LGA in Ontario, the most populous province with a high concentration of Asian population in Canada.

\section{RESEARCH DESIGN AND METHODS}

Study design and data sources

The study design is a population-based retrospective cohort. We use data obtained from the Better Outcomes
Registry \& Network (BORN) Ontario birth registry (https://www.bornontario.ca/en/about-born/), the largest and most robust perinatal dataset in Canada. The BORN registry contains maternal race and clinical data related to pregnancy and birth, including T1DM, T2DM, gestational diabetes, body mass index (BMI, weight in kilogram divided by height in meter squared), gestational weight gain, gestational age, and birth weight. Furthermore, to increase the identification of T1DM, T2DM, gestational diabetes cases, BORN records were linked to correspondence national Discharge Abstract Database (DAD), administered by the Canadian Institute for Health Information (CIHI) through healthcare number, for which BORN has a copy. ${ }^{19}$

\section{Study population}

The study population included Caucasian and Asian women who had prenatal screening and resulted in a live singleton birth in an Ontario hospital between April 2015 and March 2018. Approximately 70\% of Ontario pregnant women received prenatal screening in 2016. We excluded pregnant women with any of the following conditions: maternal race other than Asian and Caucasian, missing data of infant birth weight, gestational age at birth less than 22 weeks or greater than 42 weeks, multifetal pregnancies, pregnancies with a fetal congenital anomaly diagnosis, and a second gestation (for women who had two births in the study years).

\section{Outcome measure}

The outcome of interest is LGA neonates, which was defined as birth weight greater than 90th percentile according to sex-specific Canadian birth weight reference for singletons. ${ }^{20}$

\section{Exposure measures}

T1DM is the main exposure of interest. T1DM was primarily identified by a variable of "diabetes during pregnancy" which captures diagnosis of T1DM in BORN registry data. In addition, we linked BORN data to CIHI-DAD data to improve the ascertainment of T1DM by using maternal diagnosis codes (International Statistical Classification of Diseases and Related Health Problems, 10th Revision, Canada, E10). The diagnosis of diabetes in Ontario is based on the Diabetes Canada Clinical Practice Guidelines: fasting plasma glucose $\geq 7.0 \mathrm{mmol} / \mathrm{L}$ or glycated hemoglobin $\geq 6.5 \%$ or 2-hour plasma glucose in a $75 \mathrm{~g}$ oral glucose tolerance test $\geq 11.1 \mathrm{mmol} / \mathrm{L}$ or random plasma glucose $\geq 11.1 \mathrm{mmol} / \mathrm{L} .^{21}$ Individuals with T1DM is likely younger or lean or symptomatic hyperglycemia, especially with ketonuria or ketonemia. The autoantibody test will be used to differentiate between T1DM and T2DM. $^{21}$

\section{Covariates}

Covariates and confounders considered in this study were based on literature report and data availability, and included T2DM (yes or no), gestational diabetes (yes or no), prepregnancy BMI $\left(<25\right.$ or $\left.\geq 25 \mathrm{~kg} / \mathrm{m}^{2}\right)$, gestational 
weight gain categories (inadequate, within recommended, and excessive according to Institute of Medicine recommendation) ${ }^{22}$ maternal age at birth $(<35$ or $\geq 35$ years), parity (nulliparous or multiparous), social drug use/alcohol consumption/cigarette smoking during pregnancy (yes or no), conceived by assisted reproductive technology (yes or no), maternal mental health issues including anxiety or depression (yes or no), and a composite variable of any pre-existing maternal physical health conditions including pre-existing hypertension, pre-existing heart disease or pulmonary disease (yes or no). Because there is no record of income in BORN, we calculated the neighborhood household median income quintile (lowest, second, third, fourth, highest) by linking 2011 Canadian Census data with maternal postal code, using Statistics Canada's Postal Code Conversion File Plus (PCCF +$)^{23}$

\section{Effect modifier}

Race is considered as the moderator/effect modifier for the association between T1DM and risk of LGA. The eligible women comprise two racial groups in our study: Caucasian and Asian. Maternal race was self-reported by women and recorded by the prenatal care provider who completed the prenatal screening requisition.

\section{Statistical analysis}

We described maternal demographic characteristics and clinical factors, stratified by Caucasians and Asians. Categorical variables were described by count and per cent, and continuous variables were described by mean and SD. Rates of T1DM and LGA were compared between Caucasians and Asians. We assessed the associations between covariates and maternal race using Student's t-test for continuous data, and $\chi^{2}$ tests for categorical data.

Prior to testing the Caucasian-Asian differences in association between T1DM and LGA, we assessed the modification effect of race on the association between T1DM and LGA using Wald test. As the significant modification effect of race was detected, we primarily reported the racial-specific effect of T1DM using stratified regression analysis. Multivariable log-binomial regression models were used to estimate the adjusted relative risk (aRR) with $95 \%$ CI of T1DM on LGA, stratified by Caucasians and Asians. Since the stratified approach does not provide a test of statistical significance of the difference between the stratified RRs, we further included the interaction term of race*T1DM in one regression model to examine the racial-specific effect by using a specified level approach. ${ }^{24}$

Other factors associated with LGA including excessive gestational weight gain, T2DM, gestational diabetes, high BMI, advanced maternal age, parity, smoking, anxiety or depression, and conception with assisted reproductive technology, and pre-existing health conditions were also examined. In addition to those relevant factors, we considered a series of covariates to be potential confounders including neighborhood household median income, social drug use/alcohol consumption during pregnancy, antenatal healthcare provider and maternal hospital level of care. Confounders were identified by statistically significant and clinically relevant associations with exposure and outcome in univariate regression analysis. When conducting the adjusted regression model, if one independent variable will be treated as main exposure, other variables will be treated as covariates/confounders. Multiple imputation methods were used to account for missing data on the following covariates: maternal age at delivery $(0.1 \%$ missing $)$, neighborhood household income (5.1\% missing), parity (1.3\% missing), prepregnancy BMI (14.1\% missing), gestational weight gain (14.1\% missing), and conception type (6.8\%). Ten datasets were imputed by using the fully condition specification logistic regression method. All regression analyses were performed after missing data multiple imputations.

To estimate the relative contribution of each risk factor to LGA, we further used multivariable logistic regression models to calculate the standardized coefficient, stratified by Caucasians and Asians. In addition to T1DM, the contributions of other common risk factors were compared between Caucasian and Asian women, including gestational weight gain, high BMI, T2DM, parity, gestational diabetes, advanced maternal age, and conception with assisted reproductive technology. The absolute value of standardized regression coefficient reflected the contribution of each predictor to LGA neonates.

\section{Sensitivity analysis}

Two sensitivity analyses were conducted to examine the robustness of our main results. First, we used racialspecific birthweight curves to identify LGA neonates ${ }^{25}$ and evaluate the Caucasian-Asian differences in effect of T1DM on LGA. Second, we performed the completed cases analysis to compare the results with those obtained from missing data imputed database.

All analyses were performed using the Statistical Analysis System (SAS) for Windows, V.9.4 (SAS Institute, Cary, North Carolina, USA), with two-tailed tests and a significance level of $\mathrm{p}<0.05$.

\section{RESULTS}

A total of 232503 eligible women were included in the final analysis. Among them, $69.4 \%$ were Caucasian women and $30.6 \%$ were Asian women.

Maternal demographic and clinical characteristics stratified by Caucasian and Asian are shown in table 1 . The prevalence of T1DM was higher in Caucasian $(0.5 \%)$ than that in Asian women $(0.2 \%)(\mathrm{p}<0.001)$. The prevalence of LGA neonates was higher in Caucasian (11.0\%) than that in Asian women (5.0\%) ( $\mathrm{p}<0.001)$. Compared with Caucasian women, Asian women were more likely to have child at older age and with lower family income. Asian women were less likely to be obese, have excessive 
Table 1 Characteristics of women who were eligible for the study, stratified by Caucasian and Asian women

\begin{tabular}{|c|c|c|c|c|c|c|c|}
\hline \multirow[b]{2}{*}{ Characteristics } & \multicolumn{2}{|c|}{$\begin{array}{l}\text { Caucasian } \\
(n=161251)\end{array}$} & \multicolumn{2}{|c|}{$\begin{array}{l}\text { Asian } \\
(n=71252)\end{array}$} & \multicolumn{2}{|c|}{$\begin{array}{l}\text { Total } \\
(\mathrm{N}=232503)\end{array}$} & \multirow[b]{2}{*}{$P$ value } \\
\hline & $\mathbf{n}$ & $\%$ & $\mathrm{n}$ & $\%$ & $\mathbf{N}$ & $\%$ & \\
\hline Large-for-gestational-age neonates & 17852 & 11.1 & 3566 & 5.0 & 21418 & 9.2 & $<0.001$ \\
\hline Type 1 diabetes & 798 & 0.5 & 165 & 0.2 & 963 & 0.4 & $<0.001$ \\
\hline Maternal age at delivery (years) $($ mean $\pm S D)$ & $31.0 \pm 5.1$ & & $32.0 \pm 4.5$ & & & & \\
\hline$\leq 19$ & 2641 & 1.6 & 135 & 0.2 & 2776 & 1.2 & $<0.001$ \\
\hline $20-34$ & 118818 & 73.7 & 50423 & 70.8 & 169241 & 72.8 & \\
\hline $35-39$ & 33216 & 20.6 & 17046 & 23.9 & 50262 & 21.6 & \\
\hline$\geq 40$ & 6439 & 4.0 & 3598 & 5.0 & 10037 & 4.3 & \\
\hline Missing & 137 & 0.1 & 50 & 0.1 & 187 & 0.1 & \\
\hline \multicolumn{8}{|l|}{ Neighborhood median family income quintiles } \\
\hline Quintile 1 (lowest) & 26963 & 16.7 & 16123 & 22.6 & 43086 & 18.5 & $<0.001$ \\
\hline Quintile 2 & 26637 & 16.5 & 11093 & 15.6 & 37730 & 16.2 & \\
\hline Quintile 3 & 29476 & 18.3 & 12828 & 18.0 & 42304 & 18.2 & \\
\hline Quintile 4 & 38886 & 24.1 & 16779 & 23.5 & 55665 & 23.9 & \\
\hline Quintile 5 (highest) & 31415 & 19.5 & 10533 & 14.8 & 41948 & 18.0 & \\
\hline Missing & 7874 & 4.9 & 3896 & 5.5 & 11770 & 5.1 & \\
\hline \multicolumn{8}{|l|}{ Parity } \\
\hline 0 & 75205 & 46.6 & 30085 & 42.2 & 105290 & 45.3 & $<0.001$ \\
\hline$\geq 1$ & 84143 & 52.2 & 39946 & 56.1 & 124089 & 53.4 & \\
\hline Missing & 1903 & 1.2 & 1221 & 1.7 & 3124 & 1.3 & \\
\hline Body mass index $(\mathrm{BMI})\left(\mathrm{kg} / \mathrm{m}^{2}\right)($ mean $\pm \mathrm{SD})$ & $26.2 \pm 6.2$ & & $24.0 \pm 4.6$ & & & & \\
\hline Underweight (BMI <18.5) & 4091 & 2.5 & 3704 & 5.2 & 7795 & 3.4 & $<0.001$ \\
\hline Normal (BMI 18.5-24.9) & 69316 & 43.0 & 34196 & 48.0 & 103512 & 44.5 & \\
\hline Overweight (BMI 25.0-29.9) & 37757 & 23.4 & 14082 & 19.8 & 51839 & 22.3 & \\
\hline Obese $(\mathrm{BMI} \geq 30)$ & 30955 & 19.2 & 5569 & 7.8 & 36524 & 15.7 & \\
\hline Missing & 19132 & 11.9 & 13701 & 19.2 & 32833 & 14.1 & \\
\hline Gestational weight gain $(\mathrm{kg})($ mean $\pm S D)$ & $13.6 \pm 10.9$ & & $12.0 \pm 9$ & & & & \\
\hline Less than recommended & 40390 & 25.0 & 22396 & 31.4 & 62786 & 27.0 & $<0.001$ \\
\hline Within recommended range & 30442 & 18.9 & 15596 & 21.9 & 46038 & 19.8 & \\
\hline More than recommended & 71287 & 44.2 & 19559 & 27.5 & 90846 & 39.1 & \\
\hline Missing & 19132 & 11.9 & 13701 & 19.2 & 32833 & 14.1 & \\
\hline \multicolumn{8}{|l|}{ Conception type } \\
\hline In vitro fertilization & 3294 & 2.0 & 1250 & 1.8 & 4544 & 2.0 & $<0.001$ \\
\hline Intrauterine insemination & 3209 & 2.0 & 1108 & 1.6 & 4317 & 1.9 & \\
\hline Spontaneous conception & 143849 & 89.2 & 63911 & 89.7 & 207760 & 89.4 & \\
\hline Missing & 10899 & 6.8 & 4983 & 7.0 & 15882 & 6.8 & \\
\hline Drug use during pregnancy†‡ & 2961 & 1.8 & 145 & 0.2 & 3106 & 1.3 & $<0.001$ \\
\hline Alcohol exposure during pregnancy†‡ & 4366 & 2.7 & 587 & 0.8 & 4953 & 2.1 & $<0.001$ \\
\hline Smoking during pregnancy (any time)§ & 16719 & 10.4 & 745 & 1.0 & 17464 & 7.5 & $<0.001$ \\
\hline Pre-existing hypertension & 1430 & 0.9 & 584 & 0.8 & 2014 & 0.9 & 0.1184 \\
\hline Pre-existing type 2 diabetes & 713 & 0.4 & 651 & 0.9 & 1364 & 0.6 & $<0.001$ \\
\hline Pre-existing heart disease & 2287 & 1.4 & 327 & 0.5 & 2614 & 1.1 & $<0.001$ \\
\hline Pulmonary disease & 7501 & 4.7 & 802 & 1.1 & 8303 & 3.6 & $<0.001$ \\
\hline Anxiety $\ddagger$ & 18831 & 11.7 & 1189 & 1.7 & 20020 & 8.6 & $<0.001$ \\
\hline
\end{tabular}


Table 1 Continued

\begin{tabular}{|c|c|c|c|c|c|c|c|}
\hline \multirow[b]{2}{*}{ Characteristics } & \multicolumn{2}{|c|}{$\begin{array}{l}\text { Caucasian } \\
(n=161251)\end{array}$} & \multicolumn{2}{|c|}{$\begin{array}{l}\text { Asian } \\
(n=71252)\end{array}$} & \multicolumn{2}{|c|}{$\begin{array}{l}\text { Total } \\
(\mathrm{N}=232503)\end{array}$} & \multirow[b]{2}{*}{$P$ value } \\
\hline & $\mathbf{n}$ & $\%$ & $\mathbf{n}$ & $\%$ & $\mathbf{N}$ & $\%$ & \\
\hline 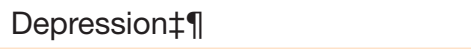 & 14520 & 9.0 & 1199 & 1.7 & 15719 & 6.8 & $<0.001$ \\
\hline Gestational diabetes & 9236 & 5.7 & 10451 & 14.7 & 19687 & 8.5 & $<0.001$ \\
\hline \multicolumn{8}{|l|}{ Antenatal healthcare provider } \\
\hline Inclusive of family physician & 17144 & 10.6 & 3509 & 4.9 & 20653 & 8.9 & $<0.001$ \\
\hline Obstetrician & 81793 & 50.7 & 55824 & 78.3 & 137617 & 59.2 & \\
\hline Family physician+obstetrician & 27403 & 17.0 & 3968 & 5.6 & 31371 & 13.5 & \\
\hline Midwife & 23168 & 14.4 & 3609 & 5.1 & 26777 & 11.5 & \\
\hline None & 184 & 0.1 & 91 & 0.1 & 275 & 0.1 & \\
\hline Other & 1987 & 1.2 & 300 & 0.4 & 2287 & 1.0 & \\
\hline Missing/unknown & 9572 & 5.9 & 3951 & 5.5 & 13523 & 5.8 & \\
\hline \multicolumn{8}{|l|}{ Maternal hospital care of level $\left.\right|^{\star \star}$} \\
\hline Level I & 17188 & 10.7 & 696 & 1.0 & 17884 & 7.7 & $<0.001$ \\
\hline Level Ila & 17485 & 10.8 & 2450 & 3.4 & 19935 & 8.6 & \\
\hline Level Ilb & 40312 & 25.0 & 22847 & 32.1 & 63159 & 27.2 & \\
\hline Level Ilc & 41975 & 26.0 & 32782 & 46.0 & 74757 & 32.2 & \\
\hline Level III & 43313 & 26.9 & 11340 & 15.9 & 54653 & 23.5 & \\
\hline Missing & 978 & 0.6 & 1137 & 1.6 & 2115 & 0.9 & \\
\hline \multicolumn{8}{|l|}{ Baby gender } \\
\hline Male & 82653 & 51.3 & 36493 & 51.2 & 119146 & 51.2 & 0.76 \\
\hline Female & 78598 & 48.7 & 34759 & 48.8 & 113357 & 48.8 & \\
\hline
\end{tabular}

Missing data were included from the percentage calculation.

*2009 Institute of Medicine recommendations.

†Drug use, alcohol use constitute any drug or alcohol use during pregnancy.

$\ddagger$ Self-reported variables.

§Maternal smoking during pregnancy captures any smoking either at the first prenatal visit or at the time of labor/admission for delivery.

IAnxiety or depression constitutes concerns during this pregnancy including those pre-existing, diagnosed during pregnancy, or active

during pregnancy.

${ }^{\star *}$ Maternal hospital level of care classification based on newborn and maternal needs, risk and illness as defined by the Provincial Council for Maternal and Child Health (Ontario).

gestational weight gain, report smoking during pregnancy, and to have mental health issues.

Figure 1 illustrates the characteristics associated with LGA, stratified by Caucasian and Asian women. In Caucasian women, T1DM (aRR 4.18, 95\% CI (3.84 to 4.55)), T2DM (aRR 1.99, 95\% CI (1.76 to 2.25)), gestational diabetes (aRR 1.44, 95\% CI (1.37 to 1.52)), high BMI $\left(>25 \mathrm{~kg} / \mathrm{m}^{2}\right)$ (aRR $1.53,95 \%$ CI (1.48 to 1.59$)$, excessive gestational weight gain (aRR 1.81, 95\% CI (1.74 to 1.89)), multiparous (aRR 1.66, 95\% CI (1.61 to 1.72)), anxiety or depression (aRR 1.06, 95\% CI (1.02 to 1.1$)$ ), preexisting health issues (aRR 1.01, 95\% CI (0.96 to 1.05)), conception with assisted reproductive technology (aRR $1.14,95 \%$ CI (1.06 to 1.22$)$ ) were identified significantly associated with increased risk of LGA neonates after adjustment for maternal demographic and clinical characteristics. However, in Asian women, T1DM (aRR 2.11, 95\% CI (1.24 to 3.59$)$ ), T2DM (aRR $2.12,95 \%$ CI (1.71 to 2.64$)$ ), gestational diabetes (aRR $1.34,95 \%$ CI (1.22 to
1.47)), high BMI $\left(>25 \mathrm{~kg} / \mathrm{m}^{2}\right)$ (aRR $1.57,95 \%$ CI $(1.45$ to 1.71)), excessive gestational weight gain (aRR 2.86, $95 \%$ CI (2.58 to 3.16$)$ ), advanced maternal age ( $>35$ years old) (aRR 1.24, 95\% CI (1.14 to 1.34)), and multiparous (aRR $1.69,95 \% \mathrm{CI}(1.55$ to 1.84$)$ ) were significantly associated with increased risk of LGA neonates (online supplemental table $\mathrm{S} 1$ ).

Table 2 shows, in overall, women with T1DM have an increased risk of LGA neonates (aRR 2.96, 95\% CI (2.27 to 3.86)), compared with women without T1DM, after adjusting for maternal age, neighborhood income level, prepregnancy BMI, gestational weight gain, parity and conception type, smoking, pre-existing health conditions, and mental health issues. Moreover, there was a significant modification effect of race on the association between T1DM and LGA neonates (Wald $\mathrm{p}<0.01$ ). The stratified regression approach (model 2) found that the association between T1DM and LGA neonates in Caucasian women (aRR 4.18, 95\% CI (3.84 to 4.55)) 


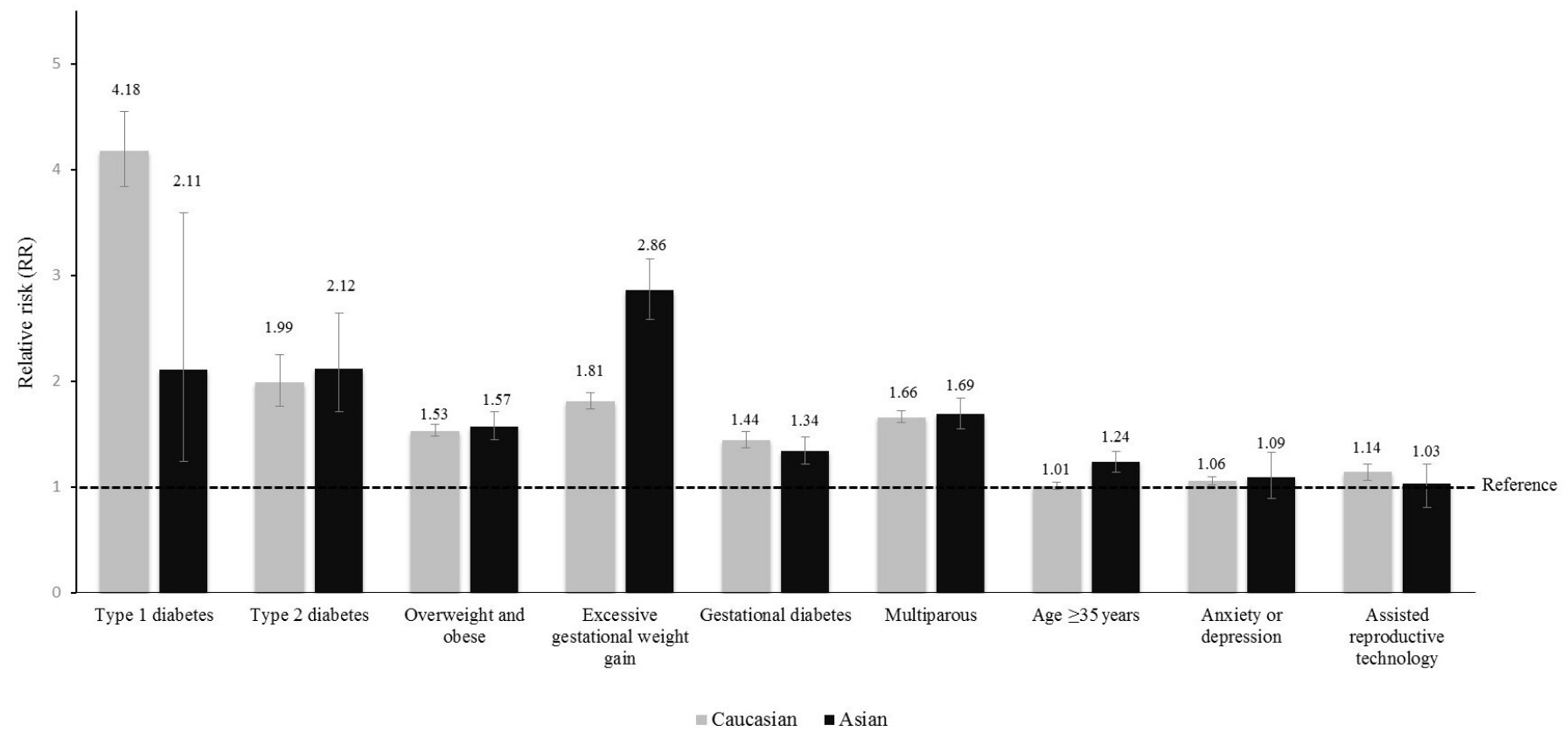

Figure 1 Relative risk of each risk factor on large-for-gestational-age neonates. Models were adjusted for maternal age, neighborhood income level, parity, smoking, prepregnancy body mass index, gestational weight gain, conception type, preexisting maternal health conditions (including pre-existing hypertension, pre-existing heart disease, or pulmonary disease), type 2 diabetes, gestational diabetes, anxiety, and depression. If one independent variable will be treated as main exposure, other factors will be treated as covariates/confounders.

was stronger than that in Asian women (aRR 2.11,95\% CI (1.23 to 3.59)). When including the interaction term of race $^{*}$ T1DM in the regression model (model 3), we found consistent results (Caucasian: aRR 4.18, 95\% CI (3.83 to 4.55); Asian: aRR 2.10, 95\% CI (1.24 to 3.54)).

Figures 2 and 3 present the standardized coefficient of each risk factor for LGA neonates among Caucasian and Asian women. T1DM was the fourth strongest contributor (standardized coefficient: 0.08 ) of LGA neonates in Caucasian women, following excessive gestational weight gain (standardized coefficient: 0.18), multiparous (standardized coefficient: 0.16), and high BMI (standardized coefficient: 0.13). However, T1DM was the seventh contributor of LGA in Asian women (standardized coefficient: 0.02), following excessive gestational weight gain (standardized coefficient: 0.25 ), multiparous (standardized coefficient: 0.15), high BMI (standardized coefficient: 0.13), gestational diabetes (standardized coefficient: 0.06 ), advanced maternal age (standardized

Table 2 Effect of T1DM on LGA neonates among Caucasian and Asian women

\begin{tabular}{|c|c|c|c|c|c|}
\hline & \multicolumn{5}{|c|}{ LGA neonates } \\
\hline & \multicolumn{2}{|c|}{$\begin{array}{l}\text { T1DM } \\
\text { (yes) }\end{array}$} & \multicolumn{2}{|l|}{$\begin{array}{l}\text { T1DM } \\
\text { (no) }\end{array}$} & \multirow{2}{*}{$\begin{array}{l}\text { Adjusted RR } \\
(95 \% \mathrm{Cl})^{\star} \dagger\end{array}$} \\
\hline & $\mathbf{n}$ & $\%$ & n & $\%$ & \\
\hline Model 1: Main effect of T1DM in whole study population & 435 & 45.2 & 20983 & 9.1 & $2.96(2.27$ to 3.86$)$ \\
\hline \multicolumn{6}{|l|}{ Model 2: Stratified regression approach } \\
\hline Effect of T1DM among Caucasian women & 314 & 52 & 14250 & 11 & 4.18 (3.84 to 4.55$)$ \\
\hline Effect of T1DM among Asian women & 13 & 14.1 & 2594 & 5.1 & $2.11(1.24$ to 3.59$)$ \\
\hline \multicolumn{6}{|c|}{ Model 3: Specified levels approach with interaction term in the model } \\
\hline Effect of T1DM among Caucasian women & 314 & 52.2 & 14250 & 11.1 & 4.18 (3.83 to 4.55$)$ \\
\hline Effect of T1DM among Asian women & 13 & 14.1 & 2594 & 5.1 & $2.10(1.24$ to 3.54$)$ \\
\hline
\end{tabular}

*Adjusted for covariates: maternal age, neighborhood income level, parity, smoking, prepregnancy BMl, gestational weight gain, conception type, pre-existing maternal health conditions (including pre-existing hypertension, pre-existing heart disease, or pulmonary disease), type 2 diabetes, gestational diabetes, anxiety, and depression.

†Multivariable log-binomial regression models were used to estimate the relative risks. Missing values of maternal age, median household income, parity and prepregnancy BMI, gestational weight gain and conception type were imputed by fully conditional specification logistic regression (a generalized logit distribution) method.

BMI, body mass index; LGA, large-for-gestational age; RR, relative risk; T1DM, type 1 diabetes mellitus. 


\section{Caucasian}

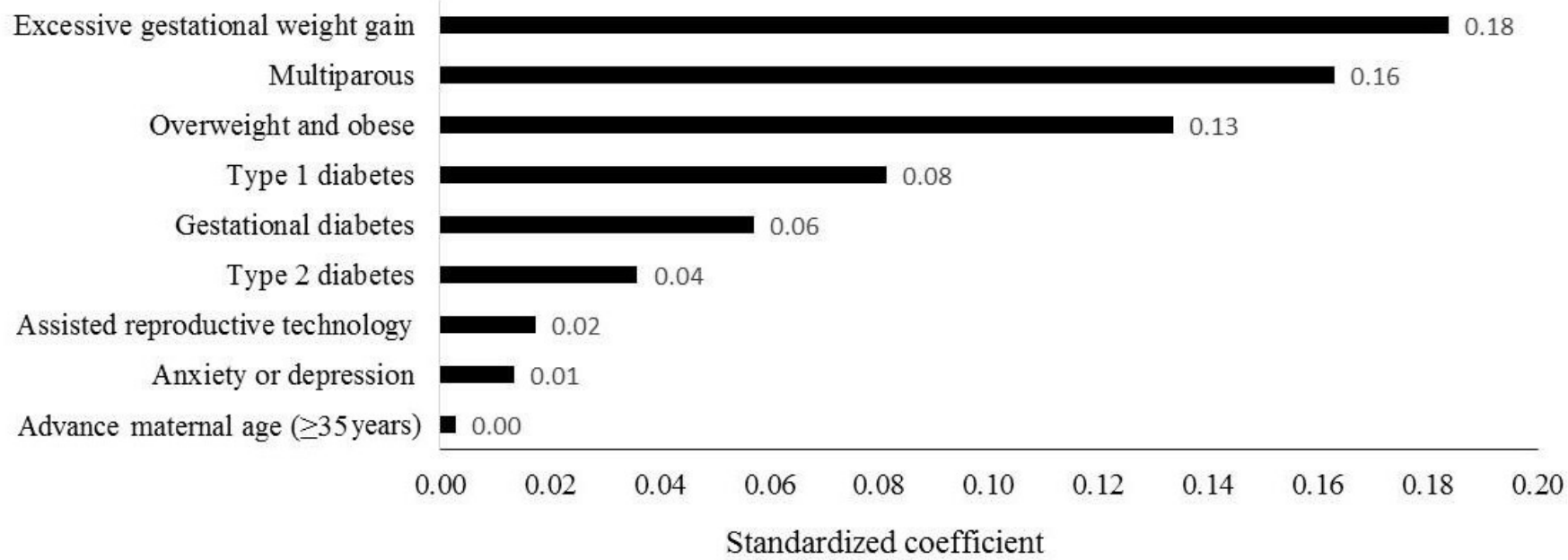

Figure 2 Contribution (standard coefficient) of each risk factor to large-for-gestational-age neonates among Caucasian women.

coefficient: 0.06), and T2DM (standardized coefficient: $0.05)$.

Sensitivity analyses found similar results with completed case analysis (online supplemental table S2). When using racial-specific birth weight reference to identify LGA neonates, the significant effect of T1DM on LGA in Asian women disappeared, but remained significant for Caucasian-Asian differences in the effect of T1DM on LGA neonates (Caucasian: aRR 4.83, 95\% CI (4.40 to 5.30); Asian: aRR $1.47,95 \%$ CI (0.89 to 2.41$)$ ) (online supplemental table S3).

\section{DISCUSSION}

In this large, population-based cohort study in the Canadian province of Ontario, we find that rates of T1DM and
LGA in Caucasians were two times of that in Asians, and the relative risk of T1DM on LGA was significantly higher in Caucasians than that in Asians. The contribution of T1DM to LGA ranked fourth in Caucasians while ranked seventh in Asians.

To our knowledge, this is the first study examining the Caucasian-Asian differences in the association between T1DM and LGA. According to the 2016 Canada Census data, Asians were the largest and fastest-growing visible minority group, accounting for $17.7 \%$ Canada's total population and $23.4 \%$ in Ontario. ${ }^{26}$ Using a contemporary and population-based registry with a robust modeling strategy and adjusting rich important confounders resulted in a stable estimated association between T1DM and LGA in each race group. Our study

Asian

Excessive gestational weight gain Multiparous

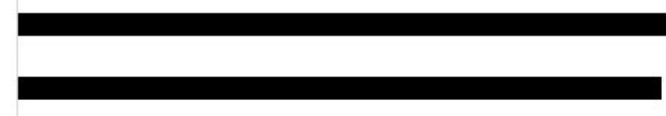

0.15

Overweight and obese

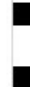

0.13

Gestational diabetes

Advance maternal age $(\geq 35$ years $)$

0.06

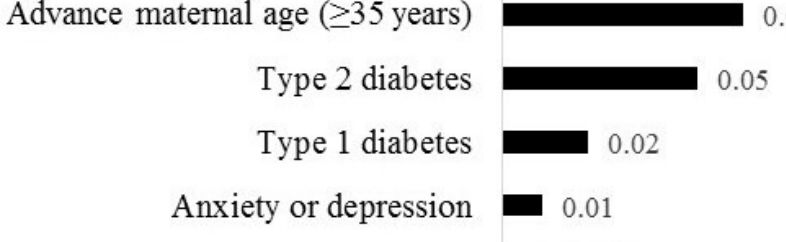

Assisted reproductive technology | -0.00022

\begin{tabular}{llllllll}
\hline-0.05 & 0 & 0.05 & 0.1 & 0.15 & 0.2 & 0.25 & 0.3
\end{tabular}

Standardized coefficient

Figure 3 Contribution (standard coefficient) of each risk factor to large-for-gestational-age neonates among Asian women. 
has a large sample size of Asian women, enabling a robust comparison of the role of T1DM on LGA between Caucasians and Asians in the context of a universal access healthcare system. Similar comparisons may be difficult to obtain in the USA and Europe due to the relatively small proportion of Asian population and multiple health insurance providers. ${ }^{27} 28$ Our finding of a higher T1DM rate in Caucasians was similar to a national study in the USA, showing that Asians have a much lower prevalence of T1DM compared with Caucasians $(0.6 \%$ for non-Hispanic white and $0.2 \%$ for non-Hispanic Asian) ${ }^{29}$ Moreover, our findings further supported the CaucasianAsian differences in the LGA rate, which was well documented in previous studies. ${ }^{30}{ }^{31}$ In addition to assessing the modification effect of race on the association between T1DM and LGA, we quantified the relative contribution of different risk factors on LGA between Caucasians and Asians. It is interesting to find that T1DM ranked fourth-strongest contributor to LGA among Caucasians, following excessive gestational weight gain, multiparous, and high BMI. On the other hand, T1DM ranked the seventh contributor of LGA in Asian women, following excessive gestational weight gain, multiparous, high BMI, gestational diabetes, advanced maternal age, and T2DM. Our findings supported previous studies that high BMI or obesity and excessive gestational weight gain were the top two modifiable risk factors of LGA for both Caucasian and Asian women. ${ }^{31-35}$ We provided further evidence that T1DM had a more significant contribution to LGA neonates in Caucasians than Asians. Our finding also demonstrated that gestational diabetes was contributed to LGA neonates, which was consistent with the report that gestational diabetes was associated with LGA trajectory at week 20 and became significant at gestational week $28 .^{36}$

Our findings of racial variations in T1DM and significant differences in the effect of T1DM on LGA by race have raised the need for better understanding the hypothesis of unique mechanisms of the development of T1DM across different races. ${ }^{37}$ Resulting from the beta-cell destruction and absolute insulin deficiency, susceptibility to T1DM involves a strong genetic component, ${ }^{39}$ especially genes encoded HLA had the highest genetic risk for disease. Heterogeneity of T1DM has been documented in the population, where the genetic, immunologic, metabolic, and clinical presentations and outcomes may vary between Caucasian and Asian considerably. ${ }^{40}$ The racial variation of immune-genetic characteristics presented by Nobel $^{41}$ that the frequency of high-risk immune-genetic alleles, haplotypes or genotypes, and their susceptibility or protective effects for T1DM risk differed among populations including Caucasians and Asians. The protective allele for T1DM has been so far reported with a higher frequency among Asian descents $(\sim 3.5 \%)$ compared with Europeans $(0.6 \%) .{ }^{42}$ Therefore, the beta cell destruction may be less potent in Asians. The variable association of the HLA-associated phenotypes to clinical onset and severity-related characteristics of autoimmune diabetes by race/ethnicity may partially explain the discrepancies of the effect of T1DM on LGA between Caucasians and Asians. ${ }^{43}$

Given the complexities of medical and social contexts associated with race and racial bias in healthcare, understanding the role of race in the effect of T1DM on LGA is valuable to inform clinical practices on prenatal management to reduce T1DM-associated morbidity in the offspring. Some studies have shown that race is more of a social construct than a biological construct. The effect of race on health outcomes tends to diminish significantly when socioeconomic status is controlled for and, in some instances, the race effect disappears. ${ }^{44}$ However, our study of Caucasian-Asian variations in the effect of T1DM on LGA seems driven by a genetic model that the Caucasian-Asian difference is determined predominantly by biological factors. Although this study uses an extensive retrospective population-based registry with adjustment for multiple biological, care practice, and socioeconomic variables, the lack of environmental and genetic contribution measurements and their interactions will limit our explanation of the moderator effect of race on T1DM and LGA ${ }^{45}$ In addition, race was reported by the mother alone in our study, although self-reported maternal race and infant genetic ancestry are closely related. ${ }^{46}$ In general, self-reported race is most reliable and should be the preferred method. However, with the increase in the number of people that belong to multiple racial categories, it is increasingly difficult to classify individuals into one race category, which further complicates the interpretation of race effects in research studies. ${ }^{47} 48$ We were unable to differentiate subgroups of South Asian and East Asian women in BORN birth registry. Previous studies have reported that the relationships between obesity and excessive gestational weight gain differ between East and South Asian groups. ${ }^{49} 50$

Other limitations were also indicated in the current study. First, we did not have glycemic control and maternal lipid levels data available in our study. ${ }^{2}$ Second, although consistent with literature reports, events of T1DM, T2DM, gestational diabetes may have been misclassified by coding errors or missed if the hyperglycemic code was not selected as the primary diagnosis. Third, to examine the robustness of our main results, we use two birthweight references to identify LGA. However, when using racial-specific birthweight curves to LGA, the difference of LGA disappeared, but the effect of T1DM on LGA between Caucasian and Asian were still significantly different. Although we used largest Canadian birth registry for this study, due to the low prevalence of T1DM in Asian women, the effect of T1DM on LGA disappeared when using racial-specific birthweight curve to identify LGA. Further data with larger sample size are needed to validate our findings. Finally, selection bias may have resulted from exclusion of women who did not complete prenatal screening, and we do not know the direction and magnitude of such potential bias. Women who underwent prenatal screening were more likely to 
live in an urban area, receive care from an obstetrician, have a higher income, and have immigrant or refugee status. ${ }^{51}$ The results generation will be caution.

In summary, our study showed that T1DM had a much greater impact on LGA in Caucasians than Asians under universal healthcare system. Among common risk factors, T1DM appears to be a more important risk factor of LGA neonates in Caucasians than Asians. Further research is warranted to better understand the etiology of Caucasian-Asian difference in T1DM on LGA, which will not only promote future prediction and prevention of LGA but also inform the clinical management practices of patients with T1DM.

\section{Author affiliations}

${ }^{1}$ Better Outcomes Registry \& Network Ontario, Ottawa, Ontario, Canada

${ }^{2}$ Children's Hospital of Eastern Ontario Research Institute, Ottawa, Ontario, Canada ${ }^{3} \mathrm{OMNI}$ Research Group, Clinical Epidemiology Program, Ottawa Hospital Research Institute, Ottawa, Ontario, Canada

${ }^{4}$ School of Epidemiology and Public Health, University of Ottawa Faculty of Medicine, Ottawa, Ontario, Canada

${ }^{5}$ Division of Endocrinology and Metabolism, University of Toronto, Toronto, Ontario, Canada

${ }^{6}$ Lunenfeld-Tanenbaum Research Institute, Mount Sinai Hospital, Toronto, Ontario, Canada

${ }^{7}$ Department of Obstetrics, Gynecology, and Newborn Care, University of Ottawa Faculty of Medicine, Ottawa, Ontario, Canada

Contributors SWW and YG developed the study concept. YG performed the analysis and interpretation of the data and drafted the manuscript. RL, DJC, RR, MCW, and SWW participated in the interpretation of the data and critically revised the manuscript. SWW and YG are the guarantors of this work.

Funding This study was supported by a Canadian Institutes of Health Research (ClHR) grant (no: FDN-148438).

Competing interests None declared.

Patient consent for publication Not required.

Ethics approval This study obtained ethical approval from the Children's Hospital of Eastern Ontario Research Ethics Board (20/10PE) prior to the commencement of this study.

Provenance and peer review Not commissioned; externally peer reviewed.

Data availability statement The data analyzed during this study are held securely at the prescribed registry BORN Ontario. Data sharing regulations prevent these data from being made available publicly due to the personal health information in the datasets. Enquiries regarding BORN data must be directed to BORN Ontario ( Science@BORNOntario.ca).

Supplemental material This content has been supplied by the author(s). It has not been vetted by BMJ Publishing Group Limited (BMJ) and may not have been peer-reviewed. Any opinions or recommendations discussed are solely those of the author(s) and are not endorsed by BMJ. BMJ disclaims all liability and responsibility arising from any reliance placed on the content. Where the content includes any translated material, BMJ does not warrant the accuracy and reliability of the translations (including but not limited to local regulations, clinical guidelines, terminology, drug names and drug dosages), and is not responsible for any error and/or omissions arising from translation and adaptation or otherwise.

Open access This is an open access article distributed in accordance with the Creative Commons Attribution Non Commercial (CC BY-NC 4.0) license, which permits others to distribute, remix, adapt, build upon this work non-commercially, and license their derivative works on different terms, provided the original work is properly cited, appropriate credit is given, any changes made indicated, and the use is non-commercial. See: http://creativecommons.org/licenses/by-nc/4.0/.

ORCID iD

Yanfang Guo http://orcid.org/0000-0003-4749-2033
REFERENCES

1 Daneman D. Type 1 diabetes. Lancet 2006;367:847-58.

2 McGrath RT, Glastras SJ, Hocking SL, et al. Large-for-gestationalage neonates in type 1 diabetes and pregnancy: contribution of factors beyond hyperglycemia. Diabetes Care 2018;41:1821-8.

3 Jensen D, Damm P, Moelsted-Pedersen L, et al. Outcomes in type 1 tiabetic pregnancies. Diabetes Care 2004;27:2819-23.

4 Maresh MJA, Holmes VA, Patterson CC, et al. Glycemic targets in the second and third trimester of pregnancy for women with type 1 diabetes. Diabetes Care 2015;38:34-42.

5 Oken E, Gillman MW. Fetal origins of obesity. Obes Res 2003;11:496-506.

6 Maahs DM, West NA, Lawrence JM, et al. Epidemiology of type 1 diabetes. Endocrinol Metab Clin North Am 2010;39:481-97.

7 Chiang JL, Kirkman MS, Laffel LMB, et al. Type 1 diabetes through the life span: a position statement of the American diabetes association. Diabetes Care 2014;37:2034-54.

8 Simmons D. Diabetes and obesity in pregnancy. Best Pract Res Clin Obstet Gynaecol 2011;25:25-36.

9 Park Y. Why is type 1 diabetes uncommon in Asia? Ann N Y Acad Sci 2006;1079:31-40.

10 Mackin ST, Nelson SM, Kerssens JJ, et al. SDRN epidemiology group. diabetes and preg Nancy: national trends over a 15 year period. Diabetologia 2018;61:1081-8.

11 Goldstein RF, Abell SK, Ranasinha S, et al. Association of gestational weight gain with maternal and infant outcomes: a systematic review and meta-analysis. JAMA 2017;317:2207-25.

12 Persson M, Pasupathy D, Hanson U, et al. Disproportionate body composition and perinatal outcome in large-for-gestational-age infants to mothers with type 1 diabetes. BJOG 2012;119:565-72.

13 Bowers K, Laughon SK, Kiely M, et al. Gestational diabetes, pre-pregnancy obesity and pregnancy weight gain in relation to excess fetal growth: variations by race/ethnicity. Diabetologia 2013;56:1263-71.

14 Kim SY, Sharma AJ, Sappenfield W, et al. Association of maternal body mass index, excessive weight gain, and gestational diabetes mellitus with large-for-gestational-age births. Obstet Gynecol 2014;123:737-44.

15 Rewers M, Ludvigsson J. Environmental risk factors for type 1 diabetes. Lancet 2016;387:2340-8.

16 Lefebvre DE, Powell KL, Strom A, et al. Dietary proteins as environmental modifiers of type 1 diabetes mellitus. Annu Rev Nutr 2006;26:175-202.

17 Bibbò S, Dore MP, Pes GM, et al. Is there a role for gut microbiota in type 1 diabetes pathogenesis? Ann Med 2017;49:11-22.

18 Szkudelski T. The mechanism of alloxan and streptozotocin action in B cells of the rat pancreas. Physiol Res 2001;50:537-46.

19 Miao Q, Fell DB, Dunn S, et al. Agreement assessment of key maternal and newborn data elements between birth registry and clinical administrative Hospital databases in Ontario, Canada. Arch Gynecol Obstet 2019;300:135-43.

20 Kramer MS, Platt RW, Wen SW, et al. A new and improved population-based Canadian reference for birth weight for gestational age. Pediatrics 2001;108:e35-113.

21 Canadian Diabetes Association Clinical Practice Guidelines Expert Committee, Goldenberg R, Punthakee Z, Definition PZ. Definition, classification and diagnosis of diabetes, prediabetes and metabolic syndrome. Can J Diabetes 2013;37 Suppl 1:S8-11.

22 Institute of Medicine and National Research Council. Weight gain during pregnancy: reexamining the guidelines. Washington, DC: The National Academies Press, 2009.

23 Statistics Canada. Postal CodeOM conversion file plus (PCCF+) version $6 D$, reference guide. Ottawa: Minister of Industry Statistics Canada Catalogue, 2017.

24 Van Ness PH, Allore HG. Using SAS $\AA$ to investigate effect modification program on aging. Sugi 2001;31:1-10.

25 St. Michael's Hospital. Birthweight curves for newborns according to maternal ancestry. Toronto: St. Michael's Hospital, 2020. www. stmichaelshospital.com/birthweights.php

26 Statistics Canada. Immigration and Ethnocultural Diversity: Key Results from the 2016 Census. Dly 2017.

27 Platonova A, Urso G. Asian immigration to the European Union, United States and Canada: an initial comparison. J Glob Policy Gov 2013;1:143-56.

28 Acs demographic and housing estimates. U.S. census bureau, 2019 [Accessed 1 Sep 2020].

29 Xu G, Liu B, Sun Y, et al. Prevalence of diagnosed type 1 and type 2 diabetes among US adults in 2016 and 2017: population based study. BMJ 2018;362:k1497.

30 Sridhar SB, Ferrara A, Ehrlich SF, et al. Risk of large-for-gestationalage newborns in women with gestational diabetes by race and 
ethnicity and body mass index categories. Obstet Gynecol 2013;121:1255-62.

31 Guo Y, Miao Q, Huang T, et al. Racial differences in contribution of prepregnancy obesity and excessive gestational weight gain to large-for-gestational-age neonates. Int J Obes 2020;44:1521-30.

32 Ladfors L, Shaat N, Wiberg N, et al. Fetal overgrowth in women with type 1 and type 2 diabetes mellitus. PLOS One 2017:12:e0187917.

33 McDonald SD, Woolcott C, Chapinal N, et al. Interprovincial variation in pre-pregnancy body mass index and gestational weight gain and their impact on neonatal birth weight with respect to small and large for gestational age. Can J Public Health 2018;109:527-38.

34 Ferraro ZM, Barrowman N, Prud'homme D, et al. Excessive gestational weight gain predicts large for gestational age neonates independent of maternal body mass index. J Matern Fetal Neonatal Med 2012;25:538-42.

35 Kim SY, Sharma AJ, Sappenfield W, et al. Association of maternal body mass index, excessive weight gain, and gestational diabetes mellitus with Large-for-Gestational-Age births. Obstetrics \& Gynecology 2014;123:737-44.

36 Li M, Hinkle SN, Grantz KL, et al. Glycaemic status during pregnancy and longitudinal measures of fetal growth in a multi-racial US population: a prospective cohort study. Lancet Diabetes Endocrinol 2020;8:292-300

37 Tosur M, Redondo MJ. Heterogeneity of type 1 diabetes: the effect of ethnicity. Curr Diabetes Rev 2018:14:266-72.

38 Bell RA, Mayer-Davis EJ, Beyer JW, et al. Diabetes in non-Hispanic white youth: prevalence, incidence, and clinical characteristics: the search for diabetes in youth study. Diabetes Care 2009;32 Suppl 2:S102-11.

39 Redondo MJ, Jeffrey J, Fain PR, et al. Concordance for islet autoimmunity among monozygotic twins. N Engl $\mathrm{J} \mathrm{Med}$ 2008;359:2849-50.
40 American Diabetes Association. 2. classification and diagnosis of diabetes. Diabetes Care 2016;39 Suppl 1:S13-22.

41 Noble JA. Immunogenetics of type 1 diabetes: a comprehensive review. J Autoimmun 2015;64:101-12.

42 Gragert L, Madbouly A, Freeman J, et al. Six-locus high resolution HLA haplotype frequencies derived from mixed-resolution DNA typing for the entire us donor registry. Hum Immunol 2013;74:1313-20.

43 Black MH, Lawrence JM, Pihoker C, et al. Hla-Associated phenotypes in youth with autoimmune diabetes. Pediatr Diabetes 2013;14:121-8

44 Cooper R, David R. The biological concept of race and its application to public health and epidemiology. $J$ Health Polit Policy Law 1986;11:97-116.

45 Cooper RS, Freeman VL. Limitations in the use of race in the study of disease causation. J Natl Med Assoc 1999;91:379-83.

46 Lee YL, Teitelbaum S, Wolff MS, et al. Comparing genetic ancestry and self-reported race/ethnicity in a multiethnic population in New York City. J Genet 2010;89:417-23.

47 Egede LE, Race ELE. Race, ethnicity, culture, and disparities in health care. J Gen Intern Med 2006;21:667-9.

48 Anderson NB, Bulatao RA, Cohen B. Critical perspectives on racial and ethnic differences in health in late life. Washington DC: National Academies Press (US), 2004.

49 SS Y, Kwon SC, Wyatt L, et al. Weighing in on the hidden Asian American obesity epidemic. Prev Med 2015;73:6-9.

$50 \mathrm{Kim}$ SY, England L, Sappenfield W, et al. Racial/Ethnic differences in the percentage of gestational diabetes mellitus cases attributable to overweight and obesity, Florida, 2004-2007. Prev Chronic Dis 2012;9:2012.

51 Hayeems RZ, Campitelli M, Ma X, et al. Rates of prenatal screening across health care regions in Ontario, Canada: a retrospective cohort study. CMAJ Open 2015;3:E236-43. 